\title{
Etiology of phantom limb syndrome: Insights from a 3D default space consciousness model
}

\author{
Ravinder Jerath ${ }^{\mathrm{a}, *}$, Molly W. Crawford ${ }^{\mathrm{a}}$, Mike Jensen ${ }^{\mathrm{b}}$ \\ a Augusta Women's Center, Augusta, GA, USA \\ ${ }^{\mathrm{b}}$ Graduate Program in Medical Illustration, Georgia Regents University, Augusta, GA, USA
}

\section{A R T I C L E I N F O}

\section{Article history:}

Received 29 December 2014

Accepted 23 April 2015

Available online $\mathrm{xxxx}$

\begin{abstract}
A B S T R A C T
In this article, we examine phantom limb syndrome to gain insights into how the brain functions as the mind and how consciousness arises. We further explore our previously proposed consciousness model in which consciousness and body schema arise when information from throughout the body is processed by corticothalamic feedback loops and integrated by the thalamus. The parietal lobe spatially maps visual and non-visual information and the thalamus integrates and recreates this processed sensory information within a three-dimensional space termed the "3D default space." We propose that phantom limb syndrome and phantom limb pain arise when the afferent signaling from the amputated limb is lost but the neural circuits remain intact. In addition, integration of conflicting sensory information within the default 3D space and the loss of inhibitory afferent feedback to efferent motor activity from the amputated limb may underlie phantom limb pain.
\end{abstract}

(ㄷ) 2015 Elsevier Ltd. All rights reserved.

\section{Introduction}

A significant portion of current neuroscientific research is dedicated to examining how consciousness arises and how the brain works as the mind. Sensory perception and body schema, a representation of the position and shape of limbs within space [26], make up a significant portion of conscious experience and therefore are an important area to examine when studying consciousness. In our previous article, we examined studies on contralateral neglect syndrome to gain insights into consciousness and we proposed a model of consciousness which integrates and reconciles many previously proposed consciousness models. In this article, we examine studies on phantom limb sensations and phantom limb pain to gain further insights into our previously proposed consciousness model.

\section{Sensory processing within the somatosensory cortex}

Among all sensory systems, the tactile sensory system develops first. Somatosensory responses can be measured as early as 8 weeks in utero [34]. The somatosensory cortex, in the parietal lobe, is comprised of four regions known as Brodmann's areas 3a, $3 \mathrm{~b}, 1$, and 2 [52]. These areas all process tactile sensory

\footnotetext{
* Corresponding author at: Augusta Women's Center, 2100 Central Ave, Suite \#7, Augusta, GA 30904, USA. Tel.: +1 7067365378.

E-mail address: rj605r@aol.com (R. Jerath).
}

information; however areas $3 \mathrm{~b}$ and 1 contain mirrored somatic maps [52] and experiments with non-human primates have shown that these areas respond primarily to sensations from the skin, while 3a responds to proprioception, and area 2 responds to both kinds of stimuli [38]. In addition, area $3 \mathrm{~b}$ responds to stimulation of specific areas while areas 1 and 2 respond to more general stimulation. For example, $3 \mathrm{~b}$ responds to stimulation of a single finger while areas 1 and 2 respond to stimulation of multiple fingers [38]. These areas provide overlapping coverage of sensory processing, with area $3 b$ responding to stimulation of a specific area of the body and areas 1 and 2 responding to stimulation of a general area of the body [38]. The size of the area devoted to a body part, in the somatosensory cortices, does not correlate with the actual size of the body part but rather with the density of receptors in that body part [38]. For example, human lips and hands have a much larger representation within the somatosensory cortex than other areas of the body [38]. Somatosensory homunculi, cortical representations of the human body, are located in the primary somatosensory cortex, ventral posterior thalamus (VPN), periaqueductal and periventricular gray matter [36], and primary motor cortex [54].

\section{Phantom limb syndrome and phantom pain}

The term "phantom limb" was coined by Silas Weir Mitchell, a civil war surgeon, back in 1872. Mitchell described how some patients experienced an amputated limb as still present and some even experienced pain or discomfort in the phantom limb [33]. 
However, phantom sensations are not limited to amputated limbs. Phantom sensations and phantom pain have been reported from removed teeth, breasts, eyes, and many other body parts [14] but phantom limbs are the most commonly reported phantom body experience [44]. Some patients feel that they can make voluntary movements with their phantom limb [29]; however, over time many patients claim the phantom limb has become paralyzed. The eventual loss of the ability to "move" the phantom limb could be due to lack of confirmation of these movements by visual and proprioceptive senses [41]. This emphasizes the significant role of vision in body schema and how conflicting sensory information may lead to neuronal changes. A scientific survey among veterans found that $60-80 \%$ of individuals with amputations experienced phantom limb sensations, with the majority of those sensations being painful [56]. Some sources have found that as high as 95$100 \%$ of amputees experience the sensation of a phantom limb after the loss of an arm or leg [31]. Phantom limb pain (PLP) and phantom limb sensations are very common experience that affects the majority of amputee patients. Current treatments include many pharmacological approaches, mirror therapy, mental imagery training, and even surgery in severe cases [57]. Understanding the underlying mechanism of PLP would help to develop further successful treatments and would give us important insights into consciousness, sensory processing, and other chronic pain disorders.

\section{Previously proposed mechanisms of phantom limb and treatments}

There are many proposed mechanisms for phantom limb pain, involving the central or peripheral nervous systems or a combination of both [61]. In the past, the most widely accepted theory underlying phantom limbs was attributed to irritation and nerve discharges in the severed and swollen nerve endings, known as neuromas [60]. However, treatments based on this hypothesis have had little success. For example, in extreme cases where a second amputation was performed in order to shorten the stump and remove neuromas, pain increased for most patients and in some cases lead to sensations of a second phantom stump with additional pain [43].

It has also been proposed that phantom limb sensations may be the result of afferent sensory feedback no longer dampening efferent motor cortical activity and commands and PLP is likely caused by the resulting conflicting sensory information [16]. Harris compares how conflicting sensory and motor information may lead to pathological pain to how conflicting sensory information from visual and vestibular systems results in motion sickness [16]. Harris's hypothesis may be supported by studies that have found that non-amputee patients commonly experience a phantom limb after anesthetic block. For example, 95\% of patients that receive an anesthetic block of the brachial plexus for surgery experience a vivid phantom limb [30]. Interestingly, when the eyes are closed the position of the phantom limb was different from the position of the real arm; however, the phantom limb 'jumped' to the position of the real arm when the patient opened their eyes and saw the actual location of their arm. This emphasizes the significant role of vision in the formation of body schema and illustrates how vision can overpower other senses. In addition, spinal anesthetic block of the lower body produces the experience of phantom legs in most patients [5]. These studies, in which there is loss of afferent signaling via anesthetic blockade, support the hypothesis that phantom limbs and PLP may be the result of afferent feedback no longer dampening efferent motor activity.

Today the most current widely accepted mechanism of PLP involves cortical reorganization. Many studies have shown neuroplastic changes within somatosensory and motor cortices after limb amputation. For example, Pons found that the somatosensory cortex reorganizes after the loss of sensory input [37] and some researchers have found that the somatosensory and motor cortex areas representing the amputated limb are taken over by nearby representational areas $[10,44]$. In addition, after the amputation of an arm, some patients experience sensations on the ipsilateral side of the face that feel as if they are arising from the phantom limb [44]. In fact, these sensations arise from a topographically organized map of the hand on the face that includes separate digits. The somatotopic map of the face and hand are adjacent in the somatosensory cortex therefore the sensory input of the facial area likely invades the hand area of the somatosensory cortex, resulting in stimuli on the face activating the hand region of the cortex [44]. In addition, in some patients, a topographical sensory map of the hand can also be found on the upper arm of the amputated limb [44]. This phenomenon is likely due to cross-activation of the hand area of the cortex by invasion of afferents from the upper arm [62]. This remapping phenomenon has been extensively studied in both animals and humans. For example, when Merzenich performed middle finger amputations on monkeys, he found that within 2 months the area of the cortex corresponding to the middle finger responded when adjacent fingers were touched [32]. He also found that when a digit was used excessively, the area of the cortex corresponding to that finger expanded, utilizing areas previously dedicated to adjacent fingers [32]. It wasn't until later, that this remapping phenomenon was applied to phantom limb. Although remapping and cortical reorganization likely underlies a significant portion of phantom limb sensations, it cannot explain all aspects of phantom limb [44].

A successful treatment for PLP has been the use of mirror therapy [46]. Mirror therapy consists of using a mirror to reflect the intact limb to appear in the position of the amputated limb and the patient is told to move and relax the limb [10]. This illusion makes it appear that the missing limb is moving. It is not well understood how this therapy works but a recent study found that mirror therapy reversed the dysfunctional reorganization that occurs in the somatosensory cortex [10]. The study also found that pain reduction was associated with decreased activity in the inferior parietal cortex [10]. The success of mirror therapy suggests that visual feedback can outcompete other sensory systems and can actually elicit sensory experiences from other sensory systems [24]. A study using mental imagery training in order to imagine moving and relaxing the phantom limb also found a significant reduction in PLP [27], along with elimination of cortical reorganization.

In addition to cortical reorganization, mirror neurons may also underlie the effectiveness of mirror therapy. Mirror neurons are neurons that fire both when an animal performs an action and when watching an action being performed [48]. These mirror neurons have been found in both monkeys [48] and humans [50]. In addition, during mirror therapy touching of the intact limb in the mirror elicits tactile sensations in the phantom limb [47]. Ramachandran proposes that non-amputee patients would not feel touch sensations on their intact limb during this type of exercise because signals from non-mirror touch neurons may block the mirror neuron signals and tell the brain that they are not being touched while in patients with phantom limb pain the mirror neurons are not blocked by signaling from these non-mirror touch neurons [47]. However, experiments in which subjects, without amputations, watch a rubber hand being stroked by a paintbrush report feeling the sensations on their hand that is hidden from view [4], suggesting that these mirror neurons are not in fact blocked by non-mirror neurons in healthy patients. In addition, in a study on healthy individuals the subjects 5th digit was stimulated while watching a video of the 1 st digit being stimulated 
within the peripersonal space where the hand would be [53]. This induced conflicting sensory input similar to that experienced during phantom limb sensations. When the actual stimulus was in phase with the video stimulus, the subjects felt the stimulus on the 1 st digit rather than the 5 th digit that was actually receiving the stimulus. Neuromagnetic source imaging of the primary somatosensory cortex topographical revealed that the cortical representation of the 5th digit expanded during the illusion, suggesting the somatosensory cortex can be rapidly modulated [53]. Another experiment created a rubber hand illusion with a finger cut off to simulate phantom limb sensations. 93\% of participants reported vivid sensations of the finger that was missing, $25 \%$ of those who reported feeling the finger also reported experiencing tingling or numbness, and 50\% experienced a change in the perceived size of the finger which they attributed to the way the experimenter mimed stroking [25]. Another proposed underlying hypothesis behind mirror therapy is that it reduces pain by removing conflicting sensory information that leads to the sensation of pain [22].

Other proposed theories underlying phantom limb involve the concept of body schema. Some researchers have proposed that body schema is fixed and does not change [5]; however many researchers and extensive research challenges this hypothesis. Body schema is likely modified by nerve impulses from sensory systems so it is not fixed and can be modified. For example, body schema has been shown to modify during tool use [17,55], extending the schema of the hand to include the tool being used. In addition, McGeoch and Ramachandran report an interesting case study involving a woman born with a malformed right hand consisting of three fingers and a rudimentary thumb. After a car crash, the patient's malformed hand had to be amputated and the patient experienced a phantom limb consisting of all five fingers, though some were shortened. McGeoch and Ramachandran propose that this suggests that the brain may have an innate template of a fully formed hand [28]. Interestingly, after the mirror box therapy she felt that the fingers on her phantom hand were of normal length [28], suggesting that the false visual feedback during the mirror therapy influenced other non-visual sensory systems and may have been involved in neuroplastic changes in the brain. The researchers propose that the hardwired representation of a full hand was inhibited by the sensory information from the afferents received from the malformed hand and that the subsequent amputation led to disinhibition of that full representation [28]. These studies suggest that body schema is not fixed but that there may be an innate framework for body schema within the brain that aids in formatting and mapping sensory information from throughout the body. We propose that the mechanisms underlying phantom limb sensations and PLP are a combination of both cortical reorganization and the formation of body schema.

\section{Hypothesis}

We propose that our consciousness model may explain the mechanism underlying phantom limb and PLP. Although we agree that corticothalamic processing and feedback loops are essential for consciousness, we propose that consciousness does not arise from functional hubs throughout the cortex, as some models have proposed. Instead, the thalamus is the central hub that integrates and creates an internal representation within a default 3D space in order for this information to rise to conscious awareness. We also propose that processed information from the sensory, motor, parietal, and other cortices is integrated by the thalamus and represented in this space, which forms body schema and self-awareness. This is a dynamic space and the internal representation within this space changes moment to moment as a result of constant, changing feedback and sensory information. This neural component of the 3D default space is likely formed by the periaqueductal gray, brainstem, and default mode network while the connected cells, throughout the body, form the rest of the 3D default space. The perception of this filled-in 3D default space occurs within the thalamus. The thalamus projects the internal space that extends throughout the entire body and the external space surrounding the body. Input from cells throughout the body and sensory organs are unconsciously processed and rise to conscious awareness when the thalamus fills in this 3D default space with the processed sensory information. The neural component of this space is likely formed by oscillations from the default mode network, other salient networks, and the ascending reticular activating system. This unconscious space and the intact somatosensory areas supersede the cognitive awareness of a lost limb, resulting in phantom limb syndrome and PLP. We have termed this a 3D "default" space to denote the intrinsic nature of the space and the possible involvement of the default mode network.

The default mode network and other salient mode networks, which utilize $90 \%$ of the brain's energy [39], may be involved in the formation of this 3D default space; however, further research is needed to support this hypothesis. The ascending reticular activating system (ARAS) is also involved in consciousness processes. Most signaling from the ARAS is modulated and relayed by the thalamus, in fact the thalamus can be considered to be part of the ARAS [59]. The thalamic reticular nucleus is involved in "gating" information from the ARAS and communicating between the brainstem and cortex [63]. The reticular activating system, along with the default mode network, is likely involved in creating the intrinsic baseline activity of the brain that forms the 3D default space and the framework on which neural activity builds. For example, the majority of the energy used by the brain is utilized by default mode network activity [11] and as little as .5-1\% of the brain's energy usage may be attributed to evoked activity [39]. The energy used for intrinsic brain activity suggests the significant role that baseline activity likely plays in supporting and creating a framework for neuronal processes, consciousness, and possibly a 3D default space.

Our proposed consciousness model is similar to the Global Workspace Theory in which consciousness occurs in a global workspace which is supported by unconscious processing. Consciousness is a "momentarily active, subjectively experienced" event [2] that does not occur in any one single place in the mind [3]. Baars proposes that the global workspace consists of functional hubs where consciousness can arise throughout the brain when multiple streams settle on a signal to bind and propagate [3]. Our model, involves a similar neural workspace but we propose that consciousness arises via the thalamus. Tononi and Edelman proposed a similar Dynamic Core Hypothesis in which consciousness arises from clusters of thalamocortical feedback activity that create a succession of unique but unified consciousness states that form conscious experience [58]. This is a dynamic system in which various neuronal groups join and leave processing clusters depending on signaling from external and internal stimuli [58]. Although our model also emphasizes the importance of corticothalamic processing and the dynamic nature of this processing, we emphasize the role of the thalamus as the central hub that is involved in the integration of highly processed information within the 3D default space that allows for consciousness to arise. Our model incorporates important elements from these widely accepted consciousness models into a more comprehensive mechanism involving the entire body and the internal representation of external space.

In our previous paper, we discussed insights about consciousness, body schema, and sensory processing by examining contralateral neglect syndrome. In our consciousness model, sensory information is processed by corticothalamic feedback loops and 
is integrated by the thalamus. An internal representation of this processed sensory information is then created by the thalamus within a 3D default space [18]. The most common cause of contralateral neglect is damage to the right parietal lobe, resulting in patients being unable to see objects on their left side and in many cases not being unable to feel stimuli on their left side [21]. Although there has been no damage to the eyes or afferent nerves though out the body, the sensory information from the left side does not rise to conscious awareness. We proposed that sensory information from the left side and left visual field is lost or damaged when it is sent to the damaged right parietal lobe [18]. The parietal lobe is involved in spatial mapping and spatial orientation [15] so when sensory information is sent to the damaged parietal lobe the information is not able to be located within space. The thalamus is not able to integrate this damaged information with other processed sensory information so it is not recreated within 3D default space and does not rise to conscious awareness [18]. For a detailed account of contralateral neglect syndrome and our proposed underlying consciousness mechanism see Jerath and Crawford [18]. The extensive processing of visual and non-visual sensory information and the representation of the external and internal worlds within the 3D default space of the mind can result in inconsistencies between external reality and conscious perception. We propose that our consciousness model may explain the mechanism underlying phantom limb sensations and PLP and the underlying conflicting sensory information.

Extensive unconscious processing likely underlies the etiology of phantom limb syndrome and PLP. After an amputation, visual sensory information and afferent sensory information from the amputated limb are processed by corticothalamic feedback loops and integrated by the thalamus. This sensory neural network remains intact after amputation; however, the majority of the afferent input, from the previous limb, no longer sends sensory information to the brain (Fig. 1). Though some afferent sensory information from the stump neuromas may be involved in sending pain signals that result in PLP, it is likely not the cause of the majority of the pain experienced during PLP. We propose that neural signaling leading to sensations of a phantom limb, combined with the visual sensory information that a limb is gone, leads to the integration of conflicting sensory information within the default space. After an amputation there is no neural damage to the somatosensory areas corresponding to the former limb and these neural representations of the body within the brain are sufficient to enable the experience of sensations from that limb in the absence of the actual limb itself [12] therefore the phantom limb is felt due to signaling within the brain rather than any afferent input. As proposed by Harris, the experience of PLP is likely similar to the mechanism of motion sickness, which results from the integration of conflicting visual and vestibular sensory information [16]. After an amputation, the integration of conflicting visual and non-visual sensory information, within the 3D default space, results in pain. Some studies have also provided evidence that there may be an intrinsic template for normal body schema within the brain that may contribute to the phenomenon of PL. For example, patients born without limbs can experience phantom limbs $[40,51]$ and in the previously discussed study, a woman born with

\section{Connections of the parietal and somatosensory cortex: visual fields and body space}

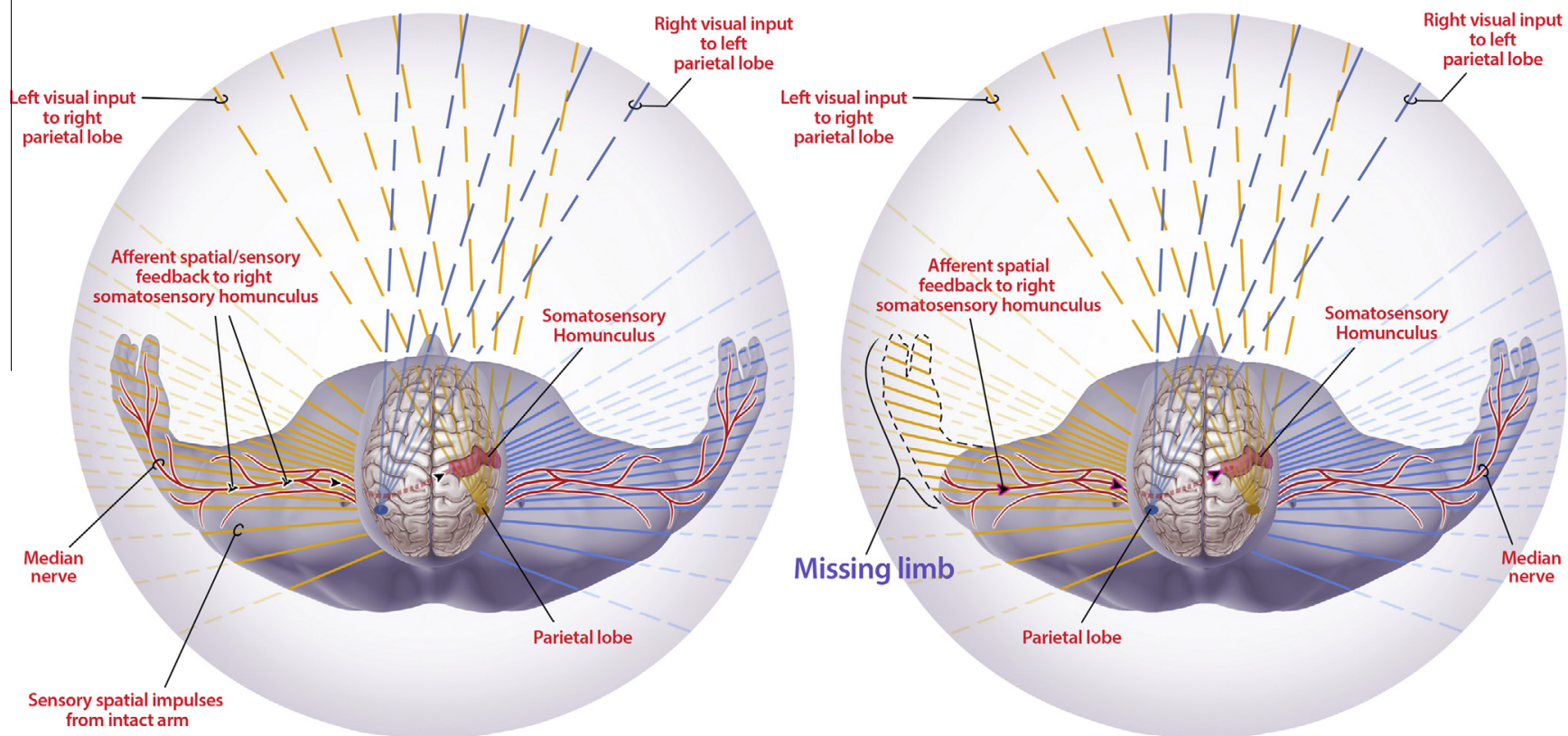

NORMAL

PHANTOM LIMB SYNDROME

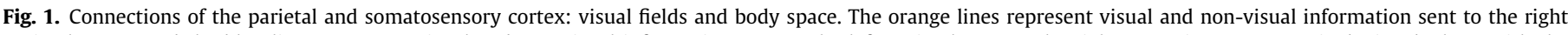

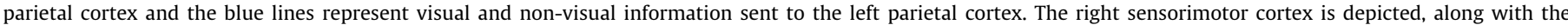

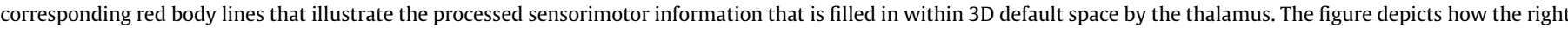

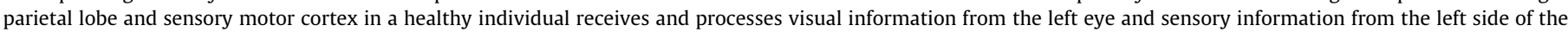

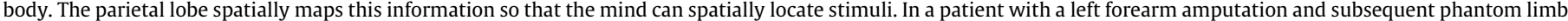

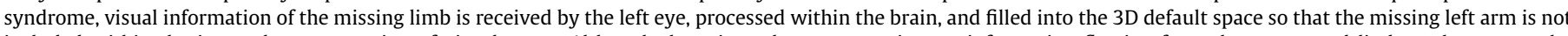

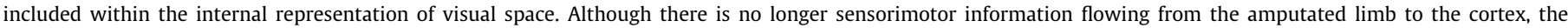

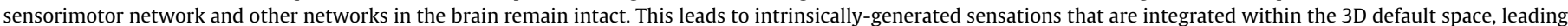

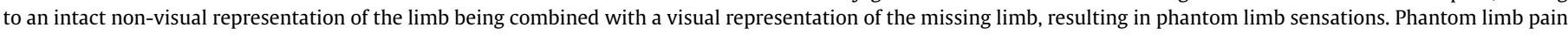

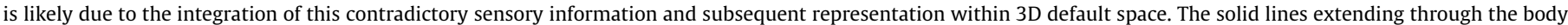

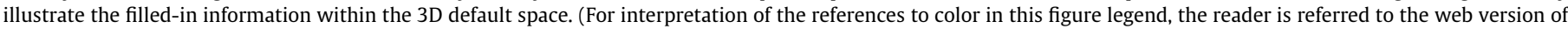
this article.) 
a malformed hand experienced a phantom limb with all five fingers after amputation [28]. This may be due to the development of neural networks and topographical sensory maps within the brain for an intact limb but the actual limb develops abnormally, resulting in phantom limb sensations that match an intact limb.

We also propose that our consciousness model likely underlies the neural mechanisms and subsequent cortical reorganization that occurs due to mirror therapy and mental imagery therapy. These two therapies likely work via a similar mechanism, with mirror therapy using visual sensory information and mental imagery therapy using imagined visual sensory information that integrates with non-visual sensory information within the 3D default space to form body schema. We propose that the surface of the skin forms the outer boundary of the 3D default space via the afferent signaling from the skin. When a person loses a limb, the neurons that correspond to that limb still form the outer boundary of a pre-existing space perceived by parietal neurons that are still active, including neurons in the somatosensory and motor cortices. All these cortical areas are involved in processing sensory information that is then integrated by the thalamus and used to fill in default space, resulting in our body schema and conscious experience. The initial amputation leads to conflicting sensory information when sensory information from the intact neural networks is integrated with visual information telling the brain the limb is missing. Following the amputation, somatosensory neurons can eventually reorganize and neurons from other somatosensory areas can invade the somatosensory area for the amputated limb leading to even further conflicting sensory information when, for example, sensory information from the hand is felt on the face (Fig. 2). This can lead to uncomfortable or unusual sensations and chronic pain. This is illustrated by studies that show that the extent of cortical reorganization correlates with the amount of pain reported by the patient $[8,20]$. This type of reorganization has also been shown to occur at the thalamic level [7]. Studies on non-human primates have also shown reorganization of sensory afferent terminations from the limb into the spinal cord and cuneate nucleus of the brainstem [9]. In addition, after mental imagery therapy or mirror therapy the subsequent reduction in pain corresponds to a reduction in cortical reorganization [27]. The reversal of dysfunctional cortical reorganization and reduction in PLP after these therapies likely involves both the activation of mirror neurons [47], the input of visual sensory information consistent with non-visual sensory information (via the mirror or mental imagery), the ability to move and relax a tensed "phantom" limb, and matching motor output with visual sensory feedback [42].

Studies on perception distortion can also give us insights into phantom limb syndrome and PLP. One study on perceptual distortion created illusory arm displacements by applying a vibrating stimulus to the tendon of the limb [35]. The study found that limb position can be distorted as easily as the perception of the location of an external object. The study also found that the internal representation of a limb's placement within space is not enough to
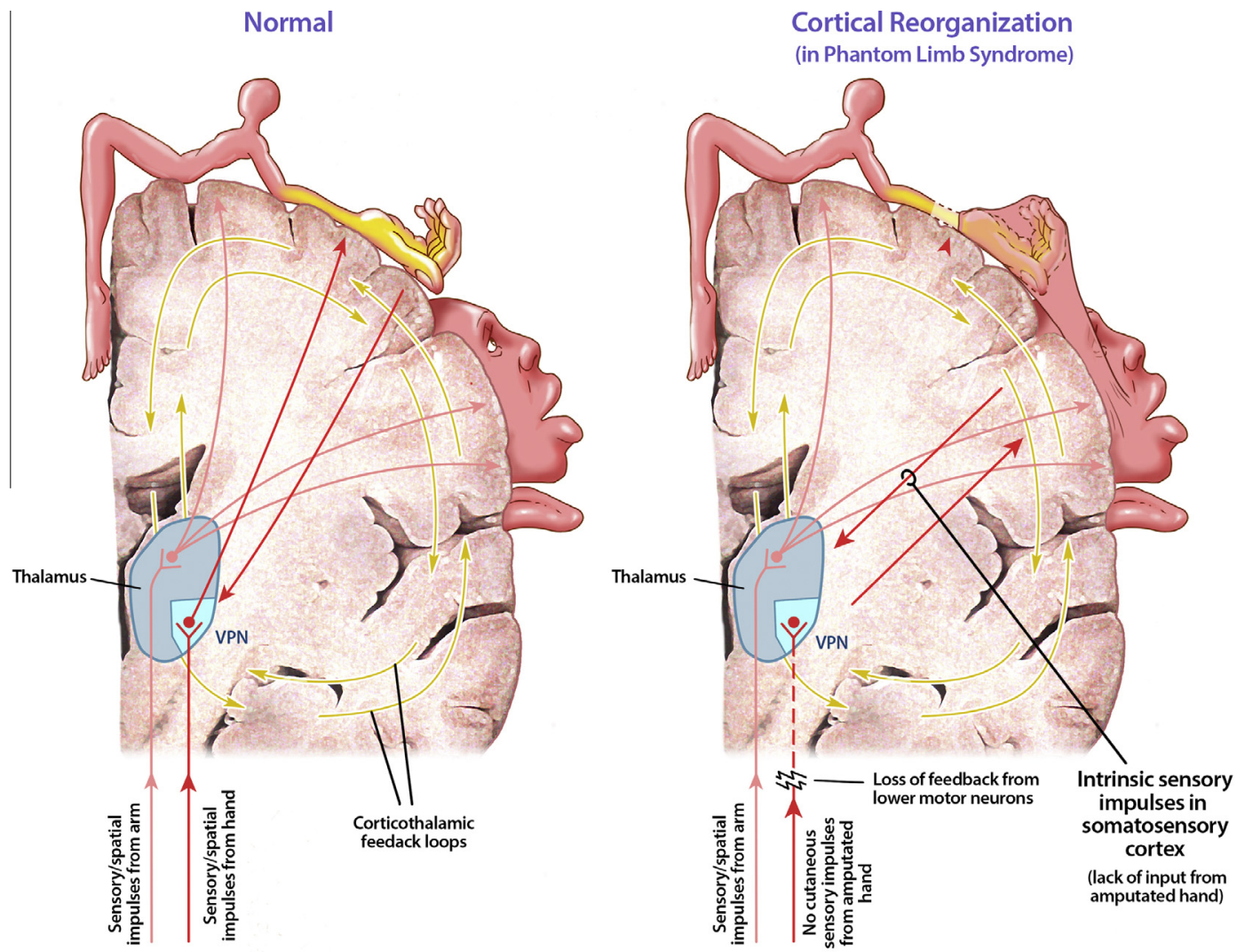

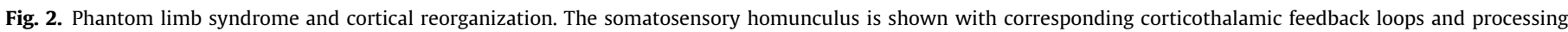

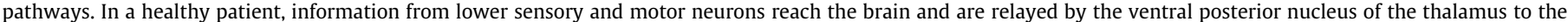

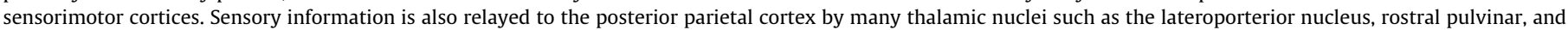

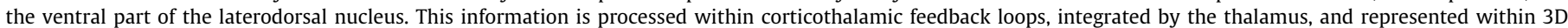

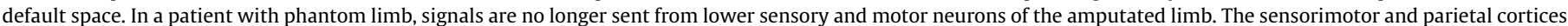

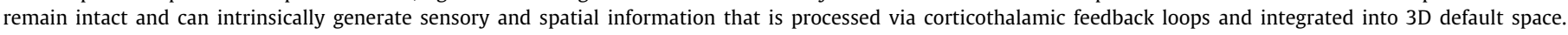

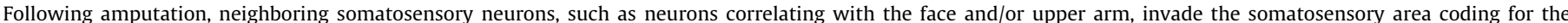

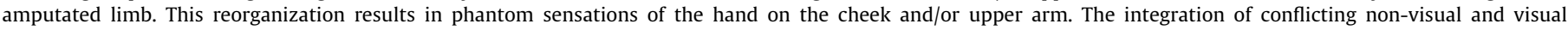
information and the subsequent representation of this information within 3D default space may underlie phantom limb pain. 
provide correct sensory information without the use of vision [35]. This may support our consciousness model by illustrating how easily perception can be distorted because it is constantly changing within the 3D default space. The study may also support a 3D default space that extends beyond the body because the perception of an external object and limb can both be easily distorted, emphasizing the unified nature of both internal and external space within the mind. The study also emphasizes the important role of vision in forming body schema and the internal representation of external and internal space. It is predictable that the visual input of a missing limb may lead to dysfunctional non-visual sensory processing. Another study, using illusory limb displacement via vibration, found that reaching and matching responses to the illusion differed, suggesting that separate body representations underlied the different action responses [19]. This supports our model in which body schema arises from constantly adjusting representations of internal and external space within the dynamic 3D default space, which would account for the separate body representations during the different experiments.

A patient who suffered a small infarct in the contralateral thalamus experienced a stinging sensation on the left side of the tongue, mouth, lips, cheeks, and the tips of the thumb and index finger on the left side. This infarct occurred in the VPN of the thalamus, which contains a somatosensory homunculus [6]. This case study is similar to a case reported by Garcin and Lapresle in which a lacune was found in the VPN of a patient who had experienced numbness of the cheek, radial edge of the forearm, and three fingers on the right side of their body [13]. In addition, a condition known as Dejerine-Roussy syndrome, thalamic pain syndrome, or central post-stroke pain can develop after a thalamic stroke [23]. This leads to a lack of sensation or tingling on the side of the body contralateral to the site of the lesion and eventually develops into severe and chronic pain [23]. Similar to PLP, this pain may be the result of the thalamic posterior insular, which contains a somatotopic pain map, responding to conflicting sensory input [45]. Cold water vestibular caloric stimulation, which activates areas in the contralateral hemisphere, has had some success in treating anosognosia, somatoparaphrenia, and contralateral neglect and may help treat thalamic pain syndrome [45] and this same treatment has been found to treat PLP [1]. One study found that after cold water vestibular stimulation, 12 out of 12 amputees that currently experienced painful phantom limbs, experienced the replacement of that phantom limb with a normal non-painful phantom limb [1]. The mechanism underlying this treatment is not well understood but because all of these disorders, that show improvement with this treatment, involve dysfunctions in bodily sensations and body schema it seems likely that this treatment works in ways other than simply causing an unconscious shift in attention. This is supported by a study that found that no shifts in attention were found in normal subjects during or after neck proprioception and cold water vestibular stimulation [49].

\section{Conclusion}

We propose a consciousness model that may underlie the phenomenon of phantom limb syndrome and phantom limb pain. Visual and non-visual sensory information is processed by corticothalamic feedback loops and integrated by the thalamus. This sensory information only rises to conscious awareness when the thalamus recreates internal and external space within the 3D default space, using this highly processed and integrated information. Studies on phantom limb syndrome and phantom limb pain have given us significant insights into sensory processing, body schema, neuroplasticity, and consciousness. We propose that in phantom limb patients the central circuits of the brain, including the somatosensory cortex and corticothalamic feedback loops, are intact while there is a loss of afferent signaling from the amputated arm, resulting in the sensation of a phantom limb. In addition, phantom limb pain likely arises from the integration of inconsistent sensory information into the body schema framework within the 3D default space which manifests as pain and discomfort. Studies have shown that visual information can overpower non-visual sensory information so that visual information, telling the brain a limb has been amputated limb, can eventually lead to a loss in previous movement of a phantom limb. This can lead to constant tension or uncomfortable positioning of the phantom limb, which contributes to phantom pain. The integration of sensory information from throughout the cortex by the thalamus, within the 3D default space, may also underlie the dysfunctional cortical reorganization that occurs after amputation and the cortical reorganization following mirror therapy.

\section{Conflict of interest statement}

None.

\section{Acknowledgement}

We would like to thank Mike Jensen MSMI, CMI of Georgia Regents University for creating the medical illustrations used in this article.

\section{References}

[1] Andre JM, Martinet N, Paysant J, Beis JM, Le Chapelain L. Temporary phantom limbs evoked by vestibular caloric stimulation in amputees. Neuropsychiatry Neuropsychol Behav Neurol 2001;14(3):190-6.

[2] Baars BJ. In the theatre of consciousness. New York NY: Oxford University Press; 1997.

[3] Baars BJ, Franklin S, Ramsøy TZ. Global workspace dynamics: cortical "binding and propagation" enables conscious contents. Front Psychol 2013;4. http:// dx.doi.org/10.3389/fpsyg.2013.00200.

[4] Botvinick M, Cohen J. Rubber hands 'feel' touch that eyes see. Nature 1998;391(6669):756. http://dx.doi.org/10.1038/35784.

[5] Bromage PR, Melzack R. Phantom limbs and the body schema. Can Anaesth Soc J 1974;21(3):267-74.

[6] Combarros O, Polo JM, Pascual J, Berciano J. Evidence of somatotopic organization of the sensory thalamus based on infarction in the nucleus ventralis posterior. Stroke 1991;22(11):1445-7.

[7] Davis KD, Kiss ZH, Luo L, Tasker RR, Lozano AM, Dostrovsky JO. Phantom sensations generated by thalamic microstimulation. Nature 1998;391(6665):385-7. http://dx.doi.org/10.1038/34905.

[8] Flor H, Nikolajsen L, Staehelin Jensen T. Phantom limb pain: a case of maladaptive CNS plasticity? Nat Rev Neurosci 2006;7(11):873-81. http:// dx.doi.org/10.1038/nrn1991.

[9] Florence SL, Kaas JH. Large-scale reorganization at multiple levels of the somatosensory pathway follows therapeutic amputation of the hand in monkeys. J Neurosci 1995;15(12):8083-95

[10] Foell J, Bekrater-Bodmann R, Diers M, Flor H. Mirror therapy for phantom limb pain: brain changes and the role of body representation. Eur J Pain 2014;18(5):729-39. http://dx.doi.org/10.1002/j.1532-2149.2013.00433.x.

[11] Fox MD, Snyder AZ, Vincent JL, Corbetta M, Van Essen DC, Raichle ME. The human brain is intrinsically organized into dynamic, anticorrelated functional networks. Proc Natl Acad Sci USA 2005;102(27):9673-8.

[12] Gallese V. The "conscious" dorsal stream: embodied simulation and its role in space and action conscious awareness. Psyche 2007;13(1):1-20.

[13] Garcin R, Lapresle J. Deuxieme observation personelle de syndrome sensitif de type thalamique et a topographie cheiro- orale par lesion localisee du thalamus. Rev Neurol 1960;103:474-81.

[14] Giummarra MJ, Gibson SJ, Georgiou-Karistianis N, Bradshaw JL. Central mechanisms in phantom limb perception: the past, present and future. Brain Res Rev 2007;54(1):219-32.

[15] Goldberg ME, Bisley JW, Powell KD, Gottlieb J. Saccades, salience and attention: the role of the lateral intraparietal area in visual behavior. Prog Brain Res 2006;155:157-75. http://dx.doi.org/10.1016/s0079-6123(06)550101.

[16] Harris AJ. Cortical origin of pathological pain. Lancet 1999;354(9188):1464-6 http://dx.doi.org/10.1016/s0140-6736(99)05003-5.

[17] Iriki A, Tanaka M, Iwamura Y. Coding of modified body schema during tool use by macaque postcentral neurones. NeuroReport 1996;7(14):2325-30. 
[18] Jerath R, Crawford MW. Neural correlates of visuospatial consciousness in 3D default space: insights from contralateral neglect syndrome. Conscious Cogn 2014;28:81-93. http://dx.doi.org/10.1016/i.concog.2014.06.008.

[19] Kammers MP, van der Ham IJ, Dijkerman HC. Dissociating body representations in healthy individuals: differential effects of a kinaesthetic illusion on perception and action. Neuropsychologia 2006;44(12):2430-6. http://dx.doi.org/10.1016/i.neuropsychologia.2006.04.009.

[20] Karl A, Birbaumer N, Lutzenberger W, Cohen LG, Flor H. Reorganization of motor and somatosensory cortex in upper extremity amputees with phantom limb pain. J Neurosci 2001;21(10):3609-18.

[21] Kerkhoff G. Spatial hemineglect in humans. Prog Neurobiol 2001;63(1):1-27.

[22] Kim SY, Kim YY. Mirror therapy for phantom limb pain. Korean J Pain 2012;25(4):272-4. http://dx.doi.org/10.3344/kjp.2012.25.4.272.

[23] Klit H, Finnerup NB, Jensen TS. Central post-stroke pain: clinical characteristics, pathophysiology, and management. Lancet Neurol 2009;8(9):857-68. http://dx.doi.org/10.1016/s1474-4422(09)70176-0.

[24] Knotkova H, Cruciani RA, Tronnier VM, Rasche D. Current and future options for the management of phantom-limb pain. J Pain Res 2012;5:39-49. http:/ dx.doi.org/10.2147/ipr.s16733.

[25] Lewis E, Lloyd DM, Farrell MJ. The role of the environment in eliciting phantom-like sensations in non-amputees. Front Psychol 2012;3:600. http:/ dx.doi.org/10.3389/fpsyg.2012.00600.

[26] Macaluso E, Maravita A. The representation of space near the body through touch and vision. Neuropsychologia 2010;48(3):782-95. http://dx.doi.org/ 10.1016/i.neuropsychologia.2009.10.010.

[27] MacIver K, Lloyd DM, Kelly S, Roberts N, Nurmikko T. Phantom limb pain, cortical reorganization and the therapeutic effect of mental imagery. Brain 2008;131(Pt 8):2181-91. http://dx.doi.org/10.1093/brain/awn124.

[28] McGeoch PD, Ramachandran VS. The appearance of new phantom fingers post-amputation in a phocomelus. Neurocase 2012;18(2):95-7. http:/ dx.doi.org/10.1080/13554794.2011.556128.

[29] Melzack R. Phantom limb. Sci Am 1992;266:120-6.

[30] Melzack R, Bromage PR. Experimental phantom limbs. Exp Neurol 1973;39(2):261-9. http://dx.doi.org/10.1016/0014-4886(73)90228-8.

[31] Melzack R, Katz J. Amputation and phantom limb pain. In: Ayers S, Baum A McManus C, Newman S, Wallston K, Weinman J, West R, editors. Cambridge handbook of psychology. Health and Medicine: Cambridge University Press: 2007.

[32] Merzenich MM, Nelson RJ, Stryker MP, Cynader MS, Schoppmann A, Zook JM. Somatosensory cortical map changes following digit amputation in adult monkeys. J Comp Neurol 1984;224(4):591-605. http://dx.doi.org/10.1002 cne.902240408.

[33] Mitchell SW. Injuries of nerves and their consequences. Philadelphia, PA: JB Lippincott; 1872.

[34] Montagu A. Touching: the human significance of the skin. New York: Perenniel Library; 1986.

[35] Naito E. Perceptual distortion of intrapersonal and near-personal space sensed by proprioception. Percept Mot Skills 2002;94(2):499-505. http://dx.doi.org/ 10.2466/pms.2002.94.2.499.

[36] Pereira EA, Wang S, Owen SL, Aziz TZ, Green AL. Human periventricular grey somatosensory evoked potentials suggest rostrocaudally inverted somatotopy. Stereotact Funct Neurosurg 2013;91(5):290-7. http://dx.doi.org/10.1159/ 000348324.

[37] Pons TP, Garraghty PE, Ommaya AK, Kaas JH, Taub E, Mishkin M. Massive cortical reorganization after sensory deafferentation in adult macaques. Science 1991;252(5014):1857-60.

[38] Purves D, Augustine G, Fitzpatrick D, Katz L, LaMantia AS, McNamara JO, et al. The somatic sensory system neuroscience. second ed. Sunderland, Massachusetts: Sinauer Associates, Inc.; 2001.

[39] Raichle ME, Snyder AZ. A default mode of brain function: a brief history of an evolving idea. Neuroimage 2007;37(4):1083-90.

[40] Ramachandran VS. Behavioral and magnetoencephalographic correlates of plasticity in the adult human brain. Proc Natl Acad Sci USA 1993;90(22):10413-20.
[41] Ramachandran VS. Consciousness and body image: lessons from phantom limbs, Capgras syndrome and pain asymbolia. Philos Trans R Soc Lond B Biol Sci 1998;353(1377):1851-9. http://dx.doi.org/10.1098/rstb.1998.0337.

[42] Ramachandran VS, Altschuler EL. The use of visual feedback, in particular mirror visual feedback, in restoring brain function. Brain 2009;132(Pt 7):1693-710. http://dx.doi.org/10.1093/brain/awp135.

[43] Ramachandran VS, Blakeslee S. Phantoms in the brain: probing the mysteries of the human mind. William Morrow \& Company; 1999.

[44] Ramachandran VS, Hirstein W. The perception of phantom limbs. The D. O. Hebb lecture. Brain 1998;121(Pt 9):1603-30.

[45] Ramachandran VS, McGeoch PD, Williams L. Can vestibular caloric stimulation be used to treat Dejerine-Roussy Syndrome? Med Hypotheses 2007;69(3):486-8. http://dx.doi.org/10.1016/i.mehy.2006.12.036.

[46] Ramachandran VS, Rogers-Ramachandran D. Synaesthesia in phantom limbs induced with mirrors. Proc Biol Sci 1996;263(1369):377-86. http://dx.doi.org/ 10.1098/rspb.1996.0058.

[47] Ramachandran VS, Rogers-Ramachandran D. Sensations referred to a patient's phantom arm from another subjects intact arm: perceptual correlates of mirror neurons. Med Hypotheses 2008;70(6):1233-4. http://dx.doi.org/ 10.1016/i.mehy.2008.01.008.

[48] Rizzolatti G, Fogassi L, Gallese V. Mirrors in the mind. Sci Am 2006;295(5):54-61.

[49] Rorden C, Karnath HO, Driver J. Do neck-proprioceptive and caloric-vestibular stimulation influence covert visual attention in normals, as they influence visual neglect? Neuropsychologia 2001;39(4):364-75.

[50] Rossi S, Tecchio F, Pasqualetti P, Ulivelli M, Pizzella V, Romani GL, et al. Somatosensory processing during movement observation in humans. Clin Neurophysiol 2002;113(1):16-24.

[51] Saadah ES, Melzack R. Phantom limb experiences in congenital limb-deficient adults. Cortex 1994;30(3):479-85.

[52] Sanchez-Panchuelo RM, Besle J, Beckett A, Bowtell R, Schluppeck D, Francis S. Within-digit functional parcellation of Brodmann areas of the human primary somatosensory cortex using functional magnetic resonance imaging at 7 tesla. J Neurosci 2012;32(45):15815-22. http://dx.doi.org/10.1523/jneurosci.250112.2012.

[53] Schaefer M, Noennig N, Heinze HJ, Rotte M. Fooling your feelings: artificially induced referred sensations are linked to a modulation of the primary somatosensory cortex. Neuroimage 2006;29(1):67-73. http://dx.doi.org/ 10.1016/j.neuroimage.2005.07.001.

[54] Schott GD. Penfield's homunculus: a note on cerebral cartography. J Neurol Neurosurg Psychiatry 1993;56(4):329-33.

[55] Serino A, Bassolino M, Farnè A, Làdavas E. Extended multisensory space in blind cane users. Psychol Sci 2007;18(7):642-8. http://dx.doi.org/10.1111/ j.1467-9280.2007.01952.x.

[56] Sherman RA, Sherman CJ, Parker L. Chronic phantom and stump pain among American veterans: results of a survey. Pain 1984;18(1):83-95.

[57] Subedi B, Grossberg GT. Phantom limb pain: mechanisms and treatment approaches. Pain Res Treat 2011;2011:864605. http://dx.doi.org/10.1155/ 2011/864605.

[58] Tononi G, Edelman GM. A universe of consciousness: how matter becomes imagination. New York, NY: Basic Books; 2000.

[59] Tononi G, Koch C. The neural correlates of consciousness: an update. Ann N Y Acad Sci 2008;1124:239-61. http://dx.doi.org/10.1196/annals.1440.004.

[60] Wall PD, Gutnick M. Ongoing activity in peripheral nerves: the physiology and pharmacology of impulses originating from a neuroma. Exp Neurol 1974;43(3):580-93.

[61] Weeks SR, Anderson-Barnes VC, Tsao JW. Phantom limb pain: theories and therapies. Neurologist 2010;16(5):277-86. http://dx.doi.org/10.1097/ NRL.0b013e3181edf128.

[62] Yang TT, Gallen C, Schwartz B, Bloom FE, Ramachandran VS, Cobb S. Sensory maps in the human brain. Nature 1994;368(6472):592-3. http://dx.doi.org/ $10.1038 / 368592 \mathrm{b0}$

[63] Young G, Pigott SE. Neurobiological basis of consciousness. Arch Neurol 1999;56(2):153-7. http://dx.doi.org/10.1001/archneur.56.2.153. 